\title{
Centipedegrass extract induces apoptosis through the activation of caspases and the downregulation of PI3K/AKT and MAPK phosphorylation in leukemia cells
}

\author{
HYOUNG-WOO BAI $^{1 *}$, SRILATHA BADABOINA ${ }^{1 *}$, CHUL-HONG PARK $^{1,2}$, \\ BO YUN CHOI ${ }^{1}$, YUN HEE NA ${ }^{1,3}$ and BYUNG YEOUP CHUNG ${ }^{1}$ \\ ${ }^{1}$ Advanced Radiation Technology Institute (ARTI), Korea Atomic Energy Research Institute (KAERI), \\ Jeongeup-si, Jeollabuk-do 580-185; ${ }^{2}$ School of Biological Sciences and Biotechnology and \\ ${ }^{3}$ Department of Molecular Medicine, Chonnam National University, Gwangju 500-757, Republic of Korea
}

Received May 30, 2014; Accepted November 17, 2014

DOI: $10.3892 / \mathrm{ijmm} .2014 .2012$

\begin{abstract}
Acute lymphoblastic leukemia (ALL), which involves the blood and bone marrow, is the most common type of cancer in children younger than 5 years of age. Previous studies have investigated the effects of centipedegrass extract (CGE), which is mainly composed of maysin and its derivatives, and have demonstrated that it has various biological activities, including antioxidant and anti-inflammatory activities, pancreatic lipase inhibitory activity, anti-adipogenic activity and insecticidal activity. To the best of our knowledge, this study is the first to investigate the anticancer effects of CGE in ALL cell lines and to elucidate the mechanisms underlying these effects. Cell viability was measured by thiazolyl blue tetrazolium blue (MTT) assay. Apoptosis, cell cycle progression and mitochondrial membrane potential $(\Delta \Psi \mathrm{m})$ were determined by flow cytometry. The effects of CGE on the phosphatidylinositol 3-kinase (PI3K)/AKT pathway and mitogen-activated protein kinases (MAPKs) were assessed by immunoblotting. PI3K, MAPK and caspase inhibitors were used to further confirm the molecular mechanisms involved. Our results clearly demonstrated that the proliferation of the ALL cells was significantly inhibited by CGE in a dose-dependent manner. Apoptosis was accompanied by the induction of significant G1 cell cycle arrest. The resulting alteration of the $\Delta \Psi \mathrm{m}$ increased the activity of caspase-3/7. The induction of apoptosis was enhanced by
\end{abstract}

Correspondence to: Dr Byung Yeoup Chung, Advanced Radiation Technology Institute (ARTI), Korea Atomic Energy Research Institute (KAERI), 29 Geumgu-gil, Jeongeup-si, Jeollabuk-do 580-185, Republic of Korea

E-mail: bychung@kaeri.re.kr

*Contributed equally

Key words: centipedegrass extract, apoptosis, acute lymphoblastic leukemia, Jurkat, CEM-CM3 cells, phosphatidylinositol 3-kinase/ AKT, mitogen-activated protein kinase, caspase the combined treatment of CGE with a PI3K inhibitor or an extracellular signal-regulated kinase (ERK) inhibitor, whereas the CGE-induced apoptosis was inhibited in the presence of caspase inhibitors, such as Z-VAD-FMK and Z-IETD-FMK. Furthermore, CGE inhibited PI3K activity by decreasing the levels of phosphorylated (p-)AKT, p-BAD, and Bcl-2 together with the levels of MAPKs, including p-ERK and p-JNK, but demonstrated no effects on p38 MAPK. Thus, our data suggest that CGE may be a novel natural compound with potential for use as an antitumor agent in ALL.

\section{Introduction}

In recent years, flavonoids have been investigated intensively for the treatment of various types of cancer and have been proven to be potent anticancer agents $(1,2)$. The therapeutic efficacy of many natural plants has already been described by practitioners of traditional medicine for several disorders. Centipedegrass [Eremochloa ophiuroides (Munro) Hack], which is native to China and Southeast Asia, is one of the most versatile medicinal herbs (3). Composition analysis by liquid chromatography-mass spectrometry (LC-MS)/MS has helped to identify several C-glycosidic flavones and phenolic constituents from centipedegrass, including maysin and its derivatives. Centipedegrass extract (CGE), used in the present study, is mainly composed of orientin, isoorientin, rhamnosylisoorientin, derhamnosylmaysin, maysin, luteolin and luteolin-6-C-bovinopyranose at the concentrations of 1.4, 28.1,63.1,49.1,97.3,34 and $85 \mu \mathrm{g} / \mathrm{mg}$ DW, respectively. Maysin and its derivatives have thus far been found only in centipedegrass, maize silk and teosinte. Centipedegrass is known to possess a wide spectrum of antibacterial, antifungal and insecticidal properties $(4,5)$, and a recent study reported that CGE exhibits anti-adipogenic activity (6). However, the anticancer effects of flavonoid-rich centipedegrass against acute lymphoblastic leukemia (ALL) are not yet known.

ALL, which involves the blood and bone marrow, is the most common type of cancer in children younger than 5 years of age (7). ALL is composed of many subtypes that represent different clinical behaviors and require different therapy schemes $(8,9)$. Family history and exposure to radiation may 
affect the risk of developing ALL (10). Treatment includes chemotherapy, steroids, radiation therapy, intensive combined treatments (including bone marrow or stem cell transplants) and growth factors. The cost for the complete treatment of a child with ALL is approximately US $\$ 100,000$ per patient (11). Therefore, the development of an effective chemotherapeutic regimen that selectively induces apoptosis in cancer cells is of great importance. Signaling pathways, such as the phosphatidylinositol 3-kinase (PI3K)/AKT and mitogen-activated protein kinase (MAPK)/extracellular signal-regulated kinase (ERK) pathways are frequently upregulated in ALL (12-14).

To maintain tissue homeostasis in multicellular organisms, cell proliferation and cell death must be regulated. This regulation may be achieved, in part, by coupling the process of cell cycle progression and apoptosis (15). Apoptosis is a highly regulated process and some of its important characteristics are DNA fragmentation, cell shrinkage, nuclear condensation, phosphatidylserine (PS) flipping from the inner to the outer leaflet of the plasma membrane and the alteration of mitochondrial membrane potential $(\Delta \Psi \mathrm{m})(16-19)$. The timing and order of cell cycle events are monitored during cell cycle checkpoints that occur at the G1/S boundary, in the S phase and during the G2/M phase (20).

The current study was designed to demonstrate the effects of CGE on human leukemia cell lines. To the best of our knowledge, our data provide the first evidence that CGE functions as a broad-range anticancer agent by effectively triggering apoptosis in human leukemia cells through a mechanism involving the regulation of PI3K/AKT and MAPKs, which in turn leads to caspase activation and apoptosis.

\section{Materials and methods}

Preparation of the CGE. The preparation of CGE was carried out as previously described (21). The dried centipedegrass (5 kg) leaves were ground in a Wiley mill and passed through a $420-\mu \mathrm{m}$ sieve. The ground sample $(1 \mathrm{~kg})$ was extracted 3 times with $80 \%$ methanol (MeOH, 100 l; Merck \& Co, Inc., Whitehouse Station, NJ, USA) for $24 \mathrm{~h}$ with constant shaking at ambient temperature in the dark. The extracts were filtered using No. 2 filter paper (Advantec Mfs Inc., Dublin, CA, USA) and concentrated in vacuo. The $\mathrm{MeOH}$ extracts were fractionated with n-hexane and ethyl acetate (EA) (Merck \& Co, Inc.). The EA extracts were concentrated in vacuo and the dried compounds were dissolved in $\mathrm{MeOH}$. The dissolved extracts in $\mathrm{MeOH}$ were diluted in $20 \% \mathrm{MeOH}$ and chromatographed over a Toyopearl HW-40C resin (Tosoh Co., Tokyo, Japan) column using $70 \% \mathrm{MeOH}$ (elution volume, $700 \mathrm{ml}$ ). The fraction was evaporated and freeze dried. The dried extracts were reconstituted in dimethylsulfoxide (DMSO) for the treatment of the cells.

Reagents and antibodies. Unless otherwise specified, all chemicals were purchased from Sigma Chemical Co. (St. Louis, MO, USA), including thiazolyl blue tetrazolium blue (MTT), Annexin V-FITC, protease inhibitor cocktail, propidium iodide (PI) and DMSO. Antibodies to Bcl-2 (no. 2870), BAX (no. 5023), Bid (no. 2002), cytochrome $c$ (no. 4247), poly(ADP-ribose) polymerase (PARP; no. 9542), caspase-3 (no. 9662), caspase-7 (no. 9492), caspase-9 (no. 9502), p-PI3K (no. 4228), p-AKT (Ser 473; no. 4058), p-AKT (Thr 308; no. 9275), AKT (no. 4691), p-BAD (Ser 136; no. 9291), HSP-60 (no. 4870) and GAPDH (no. 2118), as well as a horseradish peroxidase (HRP)-conjugated secondary antibody (no. 7074), the PI3K inhibitor, LY294002, and the ERK inhibitor, U0126, were obtained from Cell Signaling Technology (Beverly, MA, USA). Pan-caspase inhibitors (Z-IETD-FMK and Z-VAD-FMK) were purchased from R\&D Systems (Minneapolis, MN, USA). The ECL plus chemiluminescence kit was obtained from Amersham Pharmacia Biotech (Piscataway, NJ, USA). The mitochondrial dye, 3,3'-dihexyloxacarbocyanine iodide $\left[\mathrm{DiOC}_{6}(3)\right]$, was obtained from Molecular Probes (Carlsbad, CA, USA).

Cell culture and viability assay. The Jurkat (TIB-152), CEM-CM3 (TIB-195), CCRF-CEM (CCL-119) and MOLT-4 (CRL-1582) cell lines were obtained from the American Type Culture Collection (ATCC, Rockville, MD, USA) and cultured in RPMI-1640 medium supplemented with $10 \%$ heat-inactivated $\mathrm{FBS}$ and $100 \mathrm{U} / \mathrm{ml}$ penicillin at $37^{\circ} \mathrm{C}$ in a humidified $5 \% \mathrm{CO}_{2}$ incubator.

MTT was used to evaluate the viability of the cells. Briefly, the leukemia cells were plated at a density $1 \times 10^{5}$ cells/well in 96-well plates. The cells were treated with a series of concentrations $(2.5,5,10,20,40,80$ and $160 \mu \mathrm{g} / \mathrm{ml})$ of CGE. CGE was dissolved in DMSO and the final concentration of DMSO in the culture medium was $<0.05 \%$. After $24 \mathrm{~h}$ of incubation with CGE, $10 \mu \mathrm{l}$ of MTT solution $(5 \mathrm{mg} / \mathrm{ml}$ in PBS as a stock solution) were added to each well of a 96 -well plate followed by incubation for an additional $1 \mathrm{~h}$ at $37^{\circ} \mathrm{C}$. The MTT-reducing activity of the cells was measured by treating them with acidic isopropanol prior to reading at $570 \mathrm{~nm}$ using a microplate reader (Tecan Systems Inc., San Jose, CA, USA). The $\mathrm{IC}_{50}$ value was calculated using SigmaPlot 10.0 software (Systat Software Inc., San Jose, CA, USA) with the 4-parameter logistic function standard curve analysis for dose response.

Detection of apoptosis by flow cytometry. The extent of apoptosis was evaluated by flow cytometry using Annexin V-FITC. The cells were grown at a density of $1 \times 10^{6}$ cells in 6-well plates and treated with various concentrations of CGE [control (DMSO) and $2 \times \mathrm{IC}_{50}$ ] for $24 \mathrm{~h}$. Following treatment with CGE, the cells were harvested, washed with pre-chilled 1X PBS, and resuspended in $100 \mu \mathrm{l}$ of binding buffer (10 mM HEPES, $\mathrm{pH} 7.4$, $140 \mathrm{mM} \mathrm{NaCl}$, and $2.5 \mathrm{mM} \mathrm{CaCl}$ ). Subsequently, $100 \mathrm{ng} / \mathrm{ml}$ Annexin V-FITC were added and the mixture was incubated for $10-15 \mathrm{~min}$ in the dark at room temperature. PI $(20 \mu \mathrm{g} / \mathrm{ml})$ was added followed by incubation for an additional $15 \mathrm{~min}$ in the dark. A total of $400 \mu \mathrm{l}$ of binding buffer was then added and fluorescence was monitored immediately using an FC500 Flow Cytometer (Beckman Coulter, Fullerton, CA, USA). A total of 10,000 events was collected per sample. The analysis was carried out using CXP analysis software version 2.2 (Beckman Coulter) and the percentage of apoptotic cells was assessed.

Cell cycle distribution analysis. The cells were plated at a density of $1 \times 10^{6}$ cells/well in a 6-well plate. The cells were treated with various concentrations of CGE [control (DMSO), $\mathrm{IC}_{50}$ and $\left.2 \times \mathrm{IC}_{50}\right]$ for $24 \mathrm{~h}$. After $24 \mathrm{~h}$, the cells were washed twice with PBS. The cells were fixed with $70 \%$ ethanol over- 
night at $4^{\circ} \mathrm{C}$. The fixed cells were washed and resuspended in PBS containing $100 \mu \mathrm{g} / \mathrm{ml}$ RNase A and then incubated for $1 \mathrm{~h}$ at $37^{\circ} \mathrm{C}$. The cells were stained by the addition of $20 \mu \mathrm{g} / \mathrm{ml}$ PI for 15-20 min at room temperature in the dark. The DNA content of the stained cells was analyzed using an FC500 Flow Cytometer (Beckman Coulter). The data were analyzed using CXP analysis software version 2.2 (Beckman Coulter).

Determination of $\Delta \Psi$ m by $\mathrm{DiOC}_{6}(3)$. The $\Delta \Psi \mathrm{m}$ was determined using the $\operatorname{DiOC}_{6}(3)$ dye. The leukemia cells were treated with DMSO (controls) or with the indicated concentrations of CGE for $24 \mathrm{~h}$. Subsequently, the cells were washed in cold PBS, resuspended in PBS supplemented with $\mathrm{DiOC}_{6}(3)(40 \mathrm{nM})$, incubated in the dark at $37^{\circ} \mathrm{C}$ in an incubator with $5 \% \mathrm{CO}_{2}$ for $20 \mathrm{~min}$, and then immediately analyzed using an FC500 Flow Cytometer (Beckman Coulter). The data were analyzed using CXP analysis software version 2.2 (Beckman Coulter).

Caspase activity assay. The activity of caspase-3/7 was determined using the Caspase-Glo 3/7 Assay kit (Promega Corp., Madison, WI, USA) according to the manufacturer's instructions. The leukemia cells $(20,000$ cells/well) were seeded in a white 96-well plate and treated with CGE $\left(2 \times \mathrm{IC}_{50}\right.$ concentration) for 1, 3, 6, 12 and $24 \mathrm{~h}$. Caspase-Glo 3/7 reagent $(100 \mu \mathrm{l})$ was added to each well, and the 96-well plate was incubated on a rotary shaker at room temperature for $10 \mathrm{~min}$, and the luminescence was measured using a microplate reader (Tecan Systems Inc.). The quantification of caspase activity was calculated as the fold increase over the control sample. The caspase activity in the cells treated with CGE was normalized to the caspase activity of the control cells. To evaluate the caspase activity in the presence of various inhibitors, some cells were pre-incubated for $1 \mathrm{~h}$ with $50 \mu \mathrm{M}$ of caspase-3 inhibitor (Z-DEVD-FMK), $50 \mu \mathrm{M}$ of caspase-8 inhibitor (Z-IEDT-FMK), PI3K inhibitor (LY294002) or ERK inhibitor (U0126).

Preparation of the mitochondrial and cytosolic fractions. The leukemia cells $\left(1 \times 10^{6}\right.$ cells $)$ were treated with CGE ( $2 \times \mathrm{IC}_{50}$ concentration) for $1,3,6,12$ and $24 \mathrm{~h}$. The mitochondrial and cytosolic fractions were prepared using the Cytosol/Mitochondria Fractionation kit (Calbiochem, San Diego, CA, USA) according to the manufacturer's instructions. The cytosolic and mitochondrial fractions were stored at $-20^{\circ} \mathrm{C}$ for immunoblot analysis.

Immunoblot analysis. The protein concentration of the cytosolic and mitochondrial fractions was determined using the BSA method. An equal quantity of protein $(10 \mu \mathrm{g})$ was subjected to SDS-PAGE and transferred to PVDF membranes. The successful transfer of the protein was assessed by Ponceau-red staining. The membranes were blocked for $1 \mathrm{~h}$ at room temperature in Tris-buffered saline ( $\mathrm{pH}$ 7.4) containing $0.1 \%$ Tween-20 and 5\% skim milk. The blocked blots were incubated with primary antibodies overnight at $4^{\circ} \mathrm{C}$ using antibody dilutions recommended by the manufacturer. Further incubation was performed with HRP-conjugated secondary antibody and the protein expression was detected with the ECL Plus Chemiluminescence kit (Amersham Pharmacia Biotech) according to the manufacturer's instructions.
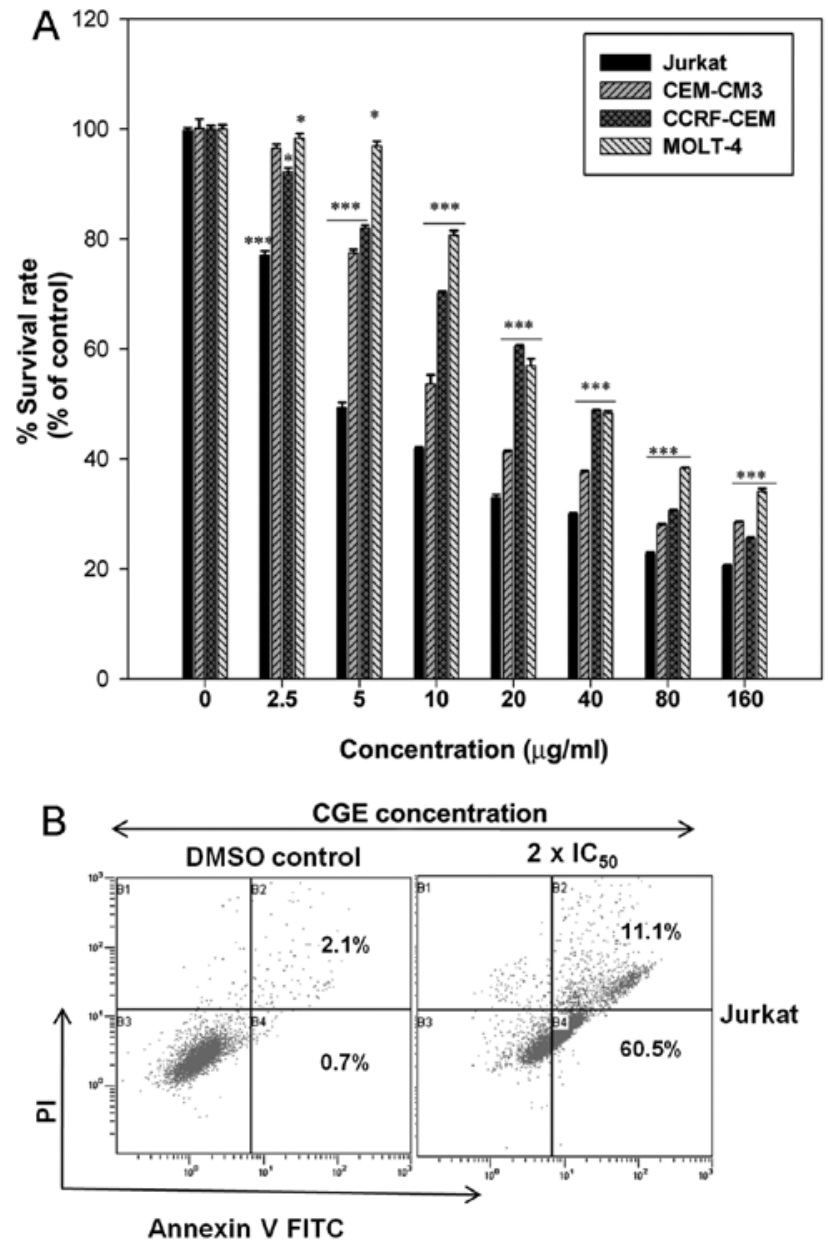

Figure 1. Effects of centipedegrass extract (CGE) on cell viability and apoptosis in leukemia cells. (A) Cell viability of Jurkat, CEM-CM3, CCRF-CEM, and MOLT-4 cells treated with various CGE concentrations as indicated for $24 \mathrm{~h}$. The cell viability was measured by an thiazolyl blue tetrazolium blue (MTT) assay. The data are presented in terms of proportional viability (\%) by comparison before and after CGE treatment, with the viability assumed to be $100 \%$. The results are presented as the means \pm SD of 3 independent experiments. The significance was determined by the Student's t-test ( ${ }^{*} \mathrm{p}<0.05$ and ${ }^{* * *} \mathrm{p}<0.001$ vs. control). (B) Cells were treated with CGE at the $2 \times \mathrm{IC}_{50}$ concentration for $24 \mathrm{~h}$ prior to flow cytometric analysis using Annexin V-FITC/propidium iodide (PI) dual staining. The x-axis indicates the number of Annexin V-FITC-labeled cells. The y-axis indicates the number of PI-labeled cells. (B4) Lower right quadrants and Annexin V-FITC-labeled cells represent the early apoptotic population. (B2) Upper right quadrants represent Annexin V-FITC/PI-dual labeled cells (late apoptotic cells).

Statistical analysis. Statistical analysis was performed by comparing the mean of the CGE-treated groups with that of the control group using an Student's unpaired t-test (Sigma Plot 10.0). Values of $\mathrm{p}<0.05, \mathrm{p}<0.01$, and $\mathrm{p}<0.001$ were considered to indicate statistically significant differences.

\section{Results}

Inhibitory effects of CGE on cell growth and the induction of apoptosis in leukemia cells. MTT assays were conducted to determine the growth inhibitory effects of CGE on leukemia cells. Fig. 1A shows the cell survival rate of the leukemia cells treated with the indicated concentrations of CGE for $24 \mathrm{~h}$. The cell survival (\%) decreased with the increasing CGE concentration. The $\mathrm{IC}_{50}$ values for CGE treatment were estimated to 

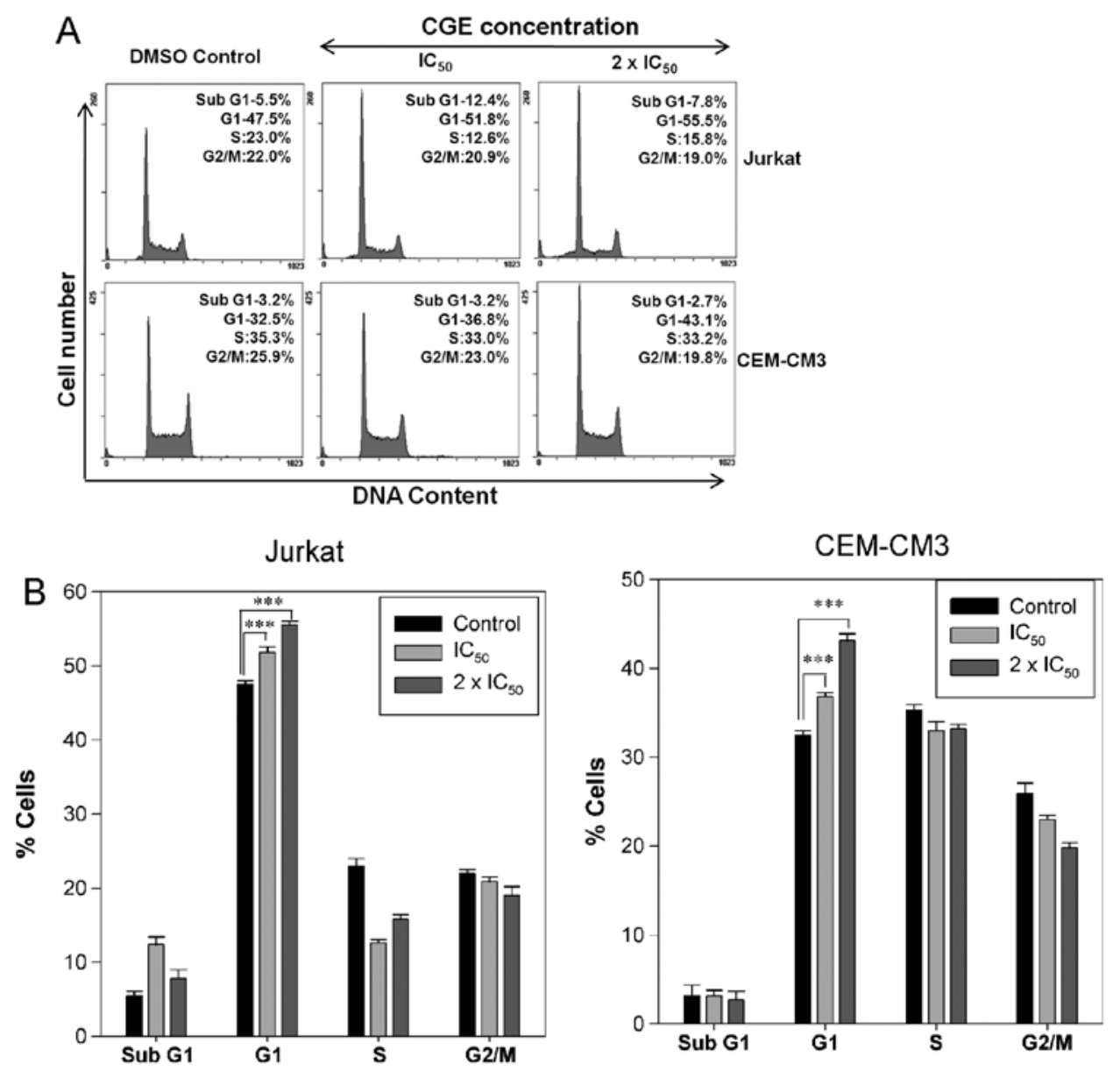

Figure 2. Centipedegrass extract (CGE) induces G1 cell cycle arrest in leukemia cells. (A) Leukemia cells were exposed to the indicated concentrations of CGE for $24 \mathrm{~h}$. Cells were fixed and labeled with propidium iodide (PI) and DNA content was analyzed by flow cytometry. (B) Data are presented as the percentage of cells in each cell cycle phase \pm SD of 3 independent experiments. ${ }^{* * *} \mathrm{p}<0.001$ vs. control.

be $3.91 \pm 0.66,7.35 \pm 0.82,43.16 \pm 2$ and $51.17 \pm 1.57 \mu \mathrm{g} / \mathrm{ml}$ for the Jurkat, CEM-CM3, CCRF-CEM and MOLT-4 cell lines, respectively, using SigmaPlot 10.0 software. These results indicate that CGE exerts a significant cytotoxic effect upon leukemia cells in a dose-dependent manner.

Due to the growth inhibitory effects observed, further experiments to determine whether CGE induces apoptosis in Jurkat cells were warranted (Fig. 1B). After treating the Jurkat cells with the $2 \times \mathrm{IC}_{50}$ concentration of CGE for $24 \mathrm{~h}$, the number of early apoptotic cells increased (60.5\%) compared with those for the DMSO control (0.7\%). The total percentage of apoptotic cells was directly related to the CGE concentration. This result is consistent with the cytotoxicity assay, and it reveals that CGE induced the apoptosis of Jurkat cells in a dose-dependent manner.

CGE induces cell cycle arrest in leukemia cells. As the Jurkat and CEM-CM3 cell lines were highly sensitive to the antiproliferation effects of CGE, the ability of CGE to interfere with the cell cycle was assessed. The Jurkat and CEM-CM3 cells were incubated with various concentrations $\left(\mathrm{IC}_{50}, 2 \times \mathrm{IC}_{50}\right)$ of CGE. Compared with the DMSO-treated controls, treatment with CGE resulted in an appreciable arrest of leukemia cells in the G1 phase of the cell cycle. The G1 phase population of Jurkat cells significantly increased from $47.5 \%$ in the control cells to 51.8 and $55.5 \%$ at the $\mathrm{IC}_{50}$ and the $2 \times \mathrm{IC}_{50}$ concentrations of CGE, respectively, whereas the G1 phase population in the CEM-CM3 control cells was $32.5 \%$ and this increased to 36.8 and $43.1 \%$ at the $\mathrm{IC}_{50}$ and the $2 \times \mathrm{IC}_{50}$ concentrations of CGE, respectively, after $24 \mathrm{~h}$ of treatment (Fig. 2A). This increase in the G1 cell population was accompanied by a decrease in cell numbers in the $S$ phase and G2/M phase in the leukemia cell lines (Fig. 2B). These results indicate that the cell cycle arrest and the induction of apoptosis may be the key mechanisms behind the antitumor activity of CGE.

CGE induces mitochondrial dysfunction. To determine whether the CGE-induced cell apoptosis is mediated through mitochondrial dysfunction, we determined the $\Delta \Psi$ m with the mitochondria-sensitive dye, $\operatorname{DiOC}_{6}(3)$, by flow cytometry. $\mathrm{DiOC}_{6}(3)$ staining was found to be increased, which indicated that CGE induced a decrease in the $\Delta \Psi \mathrm{m}$ in the leukemia cells. A percentage increase from 8.1 to $34.3 \%$ was observed in the number of Jurkat cells with a loss of $\Delta \Psi \mathrm{m}$, whereas a percentage increase from 11.5 to $25.2 \%$ was observed in the number of CEM-CM3 cells with a loss of $\Delta \Psi \mathrm{m}$ (Fig. 3). These findings strongly suggest that the CGE-induced apoptosis in leukemia cells is accompanied by breakdown of the $\Delta \Psi \mathrm{m}$. 

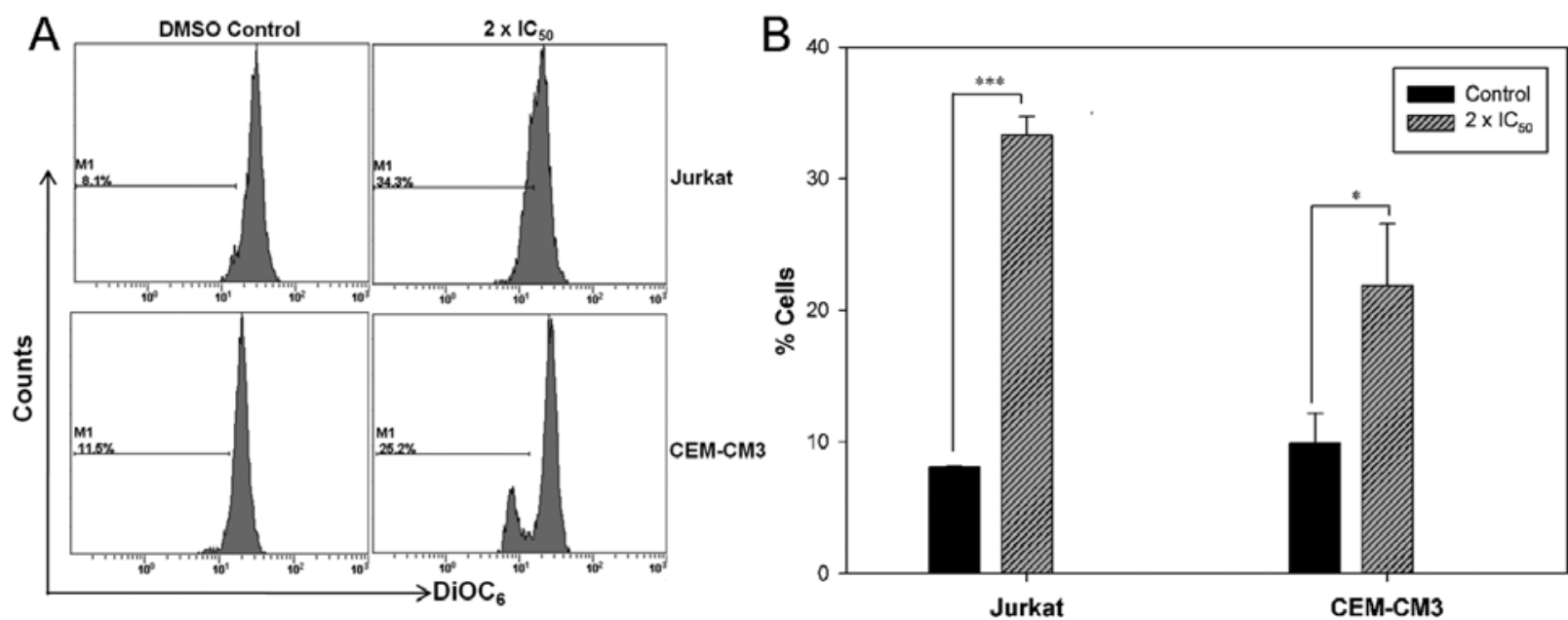

Figure 3. Centipedegrass extract (CGE) induces mitochondrial dysfunction. (A) Leukemia cells were treated with the indicated concentrations of CGE, for $24 \mathrm{~h}$ and then washed with PBS. 3,3'-Dihexyloxacarbocyanine iodide $\left[\mathrm{DiOC}_{6}(3)\right]$ staining was performed, and the mitochondrial membrane potential $(\Delta \Psi \mathrm{m})$ of the cells was analyzed by flow cytometry using the FL-1 channel. (B) Percentage of cells with loss of $\Delta \Psi \mathrm{m}$. The data are presented as the means \pm SD of 3 different experiments. ${ }^{*} \mathrm{p}<0.05$ and $^{* * *} \mathrm{p}<0.001$.

CGE-induced activation of effector caspases and cytochrome c release in leukemia cells. In order to investigate the role of caspases in apoptosis, caspase activity was analyzed after treating the leukemia cells with the $2 \times \mathrm{IC}_{50}$ concentration of CGE for 1, 3, 6, 12 and $24 \mathrm{~h}$. We observed that caspase-3/7 activity reached a maximum level (approximately 2 -fold over the control) after 12-24 h of incubation (Fig. 4A). In accordance with these results, the addition of cell permeable-specific, irreversible inhibitors of caspase-8 (Z-IETD-FMK) or caspase-3 (Z-DEVD-FMK) enzymes to the leukemia cells revealed that both the Z-IETD-FMK and Z-DEVD-FMK inhibitors completely prevented the CGE-induced activation of caspase-3/7. The CGE-induced apoptosis was inhibited in the presence of Z-VAD-FMK and Z-IETD-FMK, thereby decreasing apoptosis (Fig. 4A and C). Conversely, the combined treatment of CGE with the PI3K inhibitor, LY294002, and the ERK inhibitor, U0126, resulted in enhanced caspase activity. CGE treatment resulted in a significant increase in cleaved effector caspases (caspase-3, -7, and -9) (Fig. 4B).

In addition, we found that treatment with CGE activated caspase-3, -7 and -9 and resulted in the cleavage of the PARP substrate of caspase-3 (Fig. 4D). The effects of CGE treatment on BAX, Bid and cytochrome $c$ in the cytosolic and the mitochondrial fractions isolated from the CGE-treated leukemia cells were evident based on the low amounts of cytochrome $c$ in the cytosol after $2 \mathrm{~h}$; however, the cytochrome $c$ content markedly increased from 3 to $12 \mathrm{~h}$. A time-dependent decrease was observed in the mitochondrial fraction together with a decrease in cytosolic BAX, whereas the cleaved form of Bid and mitochondrial BAX increased, and cleaved Bid decreased in a time-dependent manner (Fig. 4D), which indicates that BAX translocation from the cytosol to the mitochondria induced CGE-mediated apoptosis.

Decreased phosphorylation of PI3K/AKT and MAPK pathway-related proteins. CGE downregulated the phosphorylation of AKT and MAPKs in the leukemia cells. We examined CGE-induced cell death by measuring the activation of apop- totic proteins in leukemia cells. The cells were treated with CGE ( $2 \times$ IC $_{50}$ concentration) for $0,1,3,6,12$ and $24 \mathrm{~h}$. CGE induced a decrease in the levels of p-PI3K, p-AKT (Ser 473), p-AKT (Thr 308), p-BAD, p-ERK and p-JNK, but the p-p38 protein levels were not altered (Fig. 5).

\section{Discussion}

The compositional analysis of CGE has demonstrated that it is composed of various $\mathrm{C}$-glycosidic flavonoids, such as maysin and its derivatives, including luteolin, orientin, isoorientin, rhamnosyl isoorientin, derhamnosly maysin and luteoin-6-C-boivinopyranose (17). CGE has been reported to exhibit several biological activities, such as pancreatic lipase inhibitory activity, antioxidant activity (4) and insecticidal activity (5). The presence of $\mathrm{C}$-glycosidic flavonoids, such as maysin and its derivatives, in CGE may attribute to the anticancer properties of CGE. Our results demonstrated that CGE inhibited cell proliferation in 4 human leukemia cell lines in a dose-dependent manner. The biochemical mechanisms through which CGE exerts its effects remain unclear, and the present study aimed to identify the molecular signaling pathways involved in the induction of apoptosis by CGE. Our results clearly demonstrated that CGE inhibited the growth of leukemia cells by inducing apoptotic cell death as determined by Annexin V-FITC analysis (Fig. 1). Generally, the early apoptotic translocation of the phospholipid PS from the inner to outer leaflet of the plasma membrane occurs due to the loss of membrane asymmetry, which can be detected by the binding of Annexin V-FITC to PS $(22,23)$.

Cell cycle control has been proven to be a major event in cellular division. The disruption of the normal cell cycle plays a vital role in the development of cancer. A large number of anticancer natural compounds have been shown to induce cell death and apoptosis in close association with cell cycle arrest at the G1 phase 9 (24-26). In this study, Jurkat and CEM-CM3 cells treated with CGE showed a significant accumulation of cells in the G1 phase; there was a concurrent reduction of 

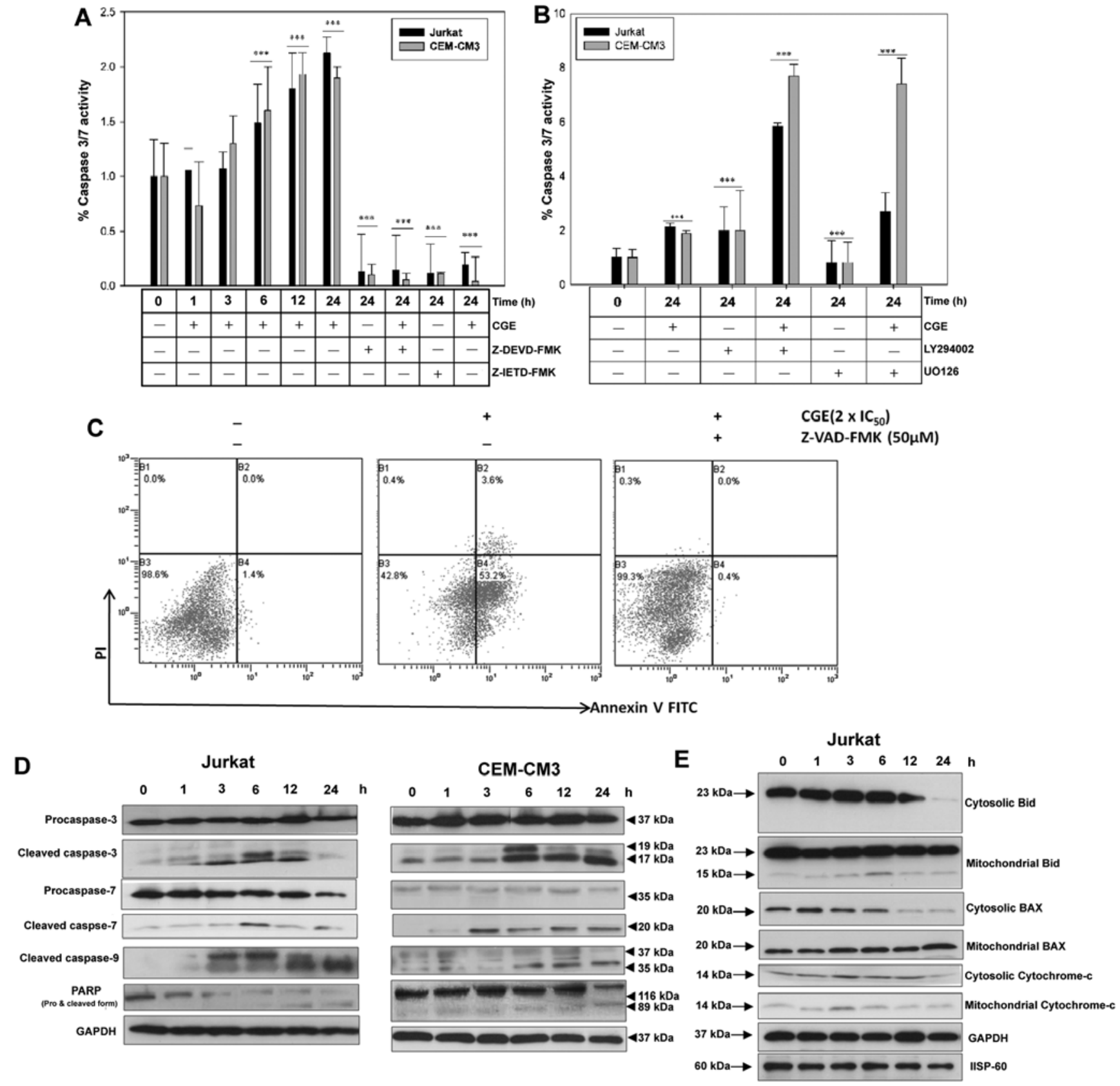

Figure 4. Jurkat and CEM-CM3 cells treated with the $2 \times \mathrm{IC}_{50}$ concentration of centipedegrass extract (CGE), for the indicated periods of time. (A) Time-dependent detection of caspase-3/7 activity in the presence or absence of specific caspase inhibitors, such as Z-IETD-FMK and Z-DEVD-FMK ( ${ }^{* * *}$ p $\left.<0.001\right)$. (B) Time-dependent detection of caspase-3/7 activity in the presence or absence of the specific phosphatidylinositol 3-kinase (PI3K) inhibitor, LY294002, and the ERK inhibitor, U0126 (**** $\mathrm{p}<0.001)$. (C) Apoptotic cell death measured by Annexin V-FITC and propidium iodide (PI) by flow cytometry. Cells were treated with CGE $\left(2 \times \mathrm{IC}_{50}\right)$ for $24 \mathrm{~h}$ in the presence or absence of a general caspase inhibitor. (D) Whole cell lysates were prepared after the cells were incubated with CGE for the indicated periods of time. Lysates were used for the detection of caspase-3, -7, -9, and poly(ADP-ribose) polymerase (PARP) in CGE-treated Jurkat and CEM-CM3 cells. GAPDH was used as an internal control. (E) The cytosolic and mitochondrial fractions were prepared as described in the Materials and methods. Cell lysates $(10 \mu \mathrm{g}$ ) were subjected to 12\% SDS-PAGE and immunoblotted with the Bid, BAX, and cytochrome $c$ antibodies. A GAPDH antibody (for the cytosolic fraction) and an HSP-60 antibody (for the mitochondrial fraction) were used as the loading controls.

the cell population in $\mathrm{S}$ and $\mathrm{G} 2 / \mathrm{M}$ phase in a dose-dependent manner (Fig. 2), which suggests that the anti-proliferative effects of CGE are mainly due to the induction of apoptosis.

There are two types of apoptotic cysteine-dependent aspartate-directed caspases: initiator and effector (executioner) caspases. Initiator caspases (e.g., caspase-8 and -9) cleave and activate effector caspases (e.g., caspase-3, -6 and -7), which in turn triggers the apoptotic process (27). Caspase- 3 has been shown to inactivate or cleave nuclear proteins, such as
PARP, which plays an important role in DNA repair (28). The initiation of this cascade reaction is regulated by caspase inhibitors, which can originate from a number of natural compounds $(29,30)$. In this study, caspase activation was examined by treating the leukemia cells with CGE. Caspase-3/7 activity increased in a time-dependent manner to approximately 2-fold over the controls after 12-24 h (Fig. 4A). Blocking caspase activation using Z-VAD-FMK and Z-IETD-FMK significantly suppressed CGE-induced apoptosis, whereas the 
A
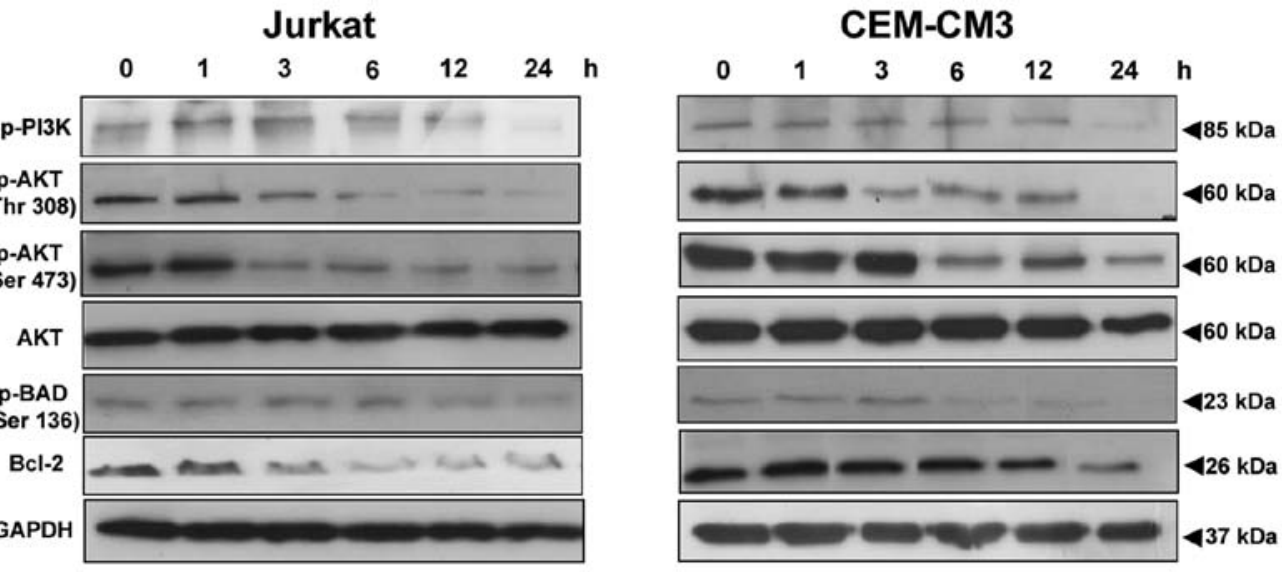

B
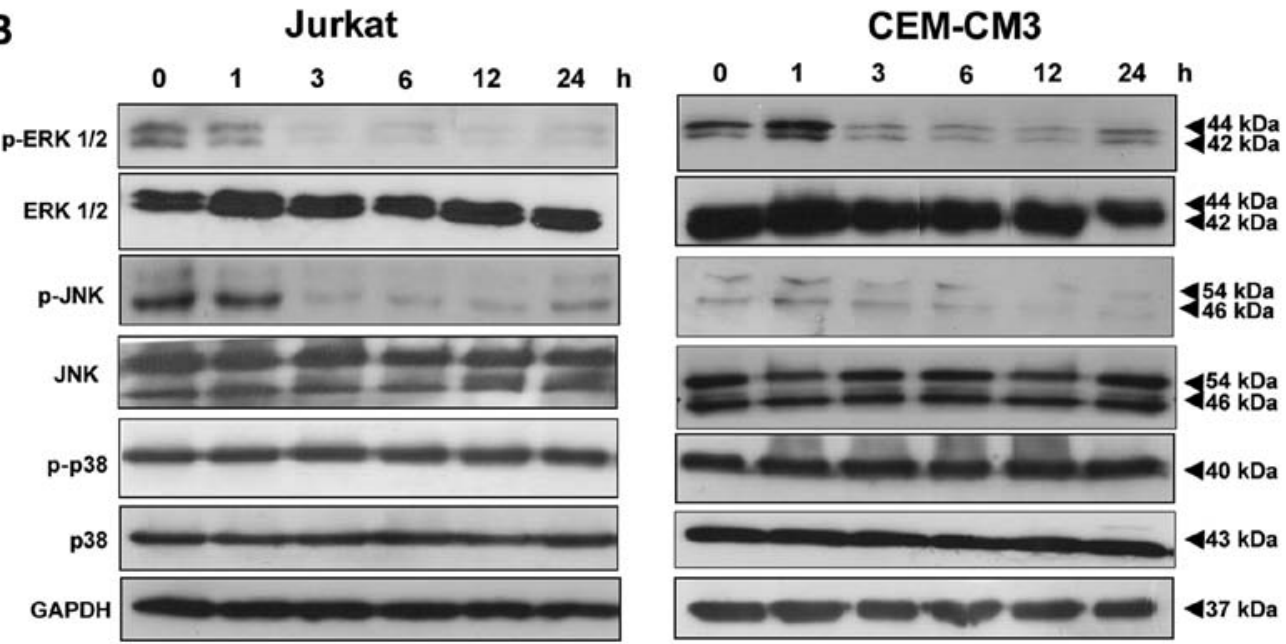

Figure 5. Effects of centipedegrass extract (CGE), on phosphatidylinositol 3-kinase (PI3K)/AKT signaling and mitogen-activated protein kinase (MAPK) phosphorylation. Cells were treated with $\mathrm{CGE}\left(2 \times \mathrm{IC}_{50}\right.$ concentration) for the indicated periods of time. (A) Whole lysates were subjected to immunoblotting for the detection of phosphorylated (p-)PI3K, p-AKT (Thr 308), p-AKT (Ser 478), AKT, p-BAD, Bcl-2 and GAPDH proteins. (B) Whole lysates were subjected to immunoblotting for MAPKs (ERK, p38, and JNK) and GAPDH as an internal control.

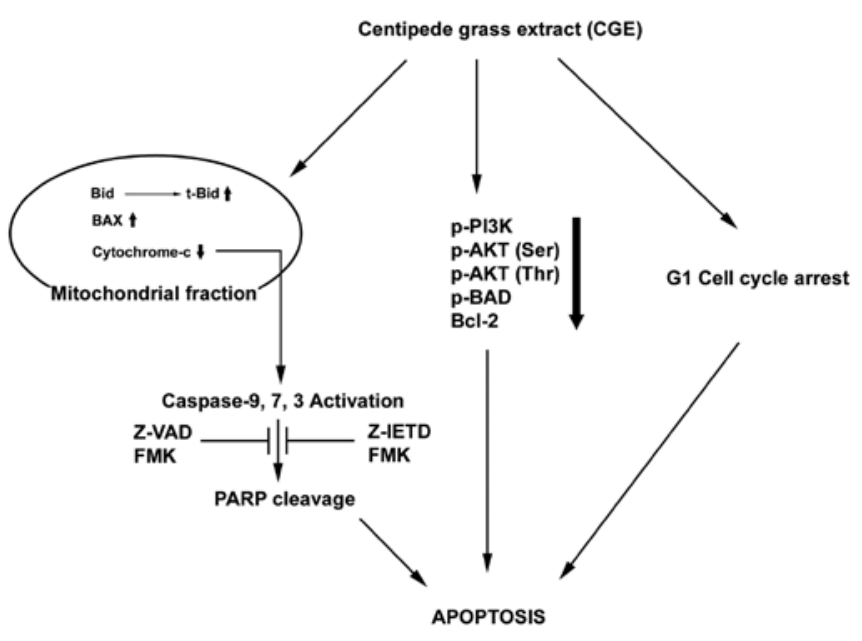

Figure 6 . Schematic diagram of the apoptotic pathway activated by centipedegrass extract (CGE) in leukemia cells.

PI3K inhibitor, LY294002, and the ERK inhibitor, U0126, enhanced the CGE-induced apoptosis (Fig. 4B) and further activated the effector caspases (caspase-3, -7, and -9) with the concomitant induction of apoptosis (Fig. 4C).
Apoptotic signaling occurs through the mitochondria and involves the cellular redistribution of BAX and cytochrome $c$ followed by the activation of multiple caspases, which manifest the apoptotic phenotype. During this process, decreased cytosolic levels of BAX, increased cytosolic levels of cytochrome $c$ and cleaved Bid are observed $(31,32)$. In this study, to better understand the cellular redistribution of proteins in CGE-induced apoptosis, we examined changes in the levels of proteins by western blot analysis. The levels of BAX decreased gradually, whereas the levels of cytochrome $c$ and cleaved Bid increased in the cytosolic fraction (Fig. 4D).

The PI3K/AKT and MAPK signaling pathways are known to function as crucial components of cell proliferation in cancer cells. Activated AKT and ERK can function as key effectors of the PI3K and MAPK signaling pathways to promote cell survival and proliferation $(33,34)$. Among the wide spectrum of proteins and genes involved in apoptosis, members of the Bcl-2 family play a central role in this process (35). The phosphorylation of Bad can block its apoptotic function (36). In addition, in this study, we observed the loss of AKT and PI3K activity and the decreased expression of p-BAD and Bcl-2, which led to apoptosis eventually.

To the best of our knowledge, this study is the first to demonstrate the anticancer effects of C-glycosidic flavonoids, such as 
maysin and its derivatives, in CGE, which inhibit the growth of leukemia cells. Our results are summarized in Fig. 6. The selective inhibition of cancer cell growth, apoptosis induction via the significant G1 cell cycle arrest and BAX translocation from the cytosol to the mitochondria induced the disruption of the $\Delta \Psi_{\mathrm{m}}$, thereby increasing the caspase-3/7 activity and activation of effector caspase-3, -7 and -9 . In addition, CGE inhibited PI3K activity by reducing p-AKT, p-BAD, and Bcl-2 levels and inhibited MAPKs, including p-ERK and p-JNK. Taken together, CGE may prove to be a potential chemopreventive agent in the treatment of ALL.

\section{Acknowledgements}

This study was supported by the National Research Foundation of Korea (NRF) grant funded by the Korean government (MSIP) (No. 2012M2A2A6036022).

\section{References}

1. Prasad S, Phromnoi K, Yadav VR, Chaturvedi MM and Aggarwal BB: Targeting inflammatory pathways by flavonoids for prevention and treatment of cancer. Planta Med 76: 1044-1063, 2010.

2. Chahar MK, Sharma N, Dobhal MP and Joshi YC: Flavonoids: a versatile source of anticancer drugs. Pharmacogn Rev 5: $1-12,2011$.

3. Wiseman BR, Gueldner RC, Lynch RE and Severson RF: Biochemical activity of centipedegrass against fall armyworm larvae. J Chem Ecol 16: 2677-2690, 1990.

4. Lee EM, Lee SS, Chung BY, et al: Pancreatic lipase inhibition by C-glycosidic flavones isolated from Eremochloa ophiuroides. Molecules 15: 8251-8259, 2010.

5. Johnson AW: Influence of organic pesticides on nematode populations and seed production of centipede grass. J Nematol 2 252-254, 1970

6. Park HJ, Chung BY, Lee MK, et al: Centipede grass exerts anti-adipogenic activity through inhibition of $\mathrm{C} / \mathrm{EBP} \beta, \mathrm{C} / \mathrm{EBP} \alpha$ and PPAR $\gamma$ expression and the AKT signaling pathway in 3T3-L1 adipocytes. BMC Complement Altern Med 12: 230, 2012.

7. Hjalgrim LL, Rostgaard K, Schmiegelow K, et al: Age- and sex-specific incidence of childhood leukemia by immuno phenotype in the Nordic countries. J Natl Cancer Inst 95: 1539-1544, 2003.

8. Yeoh EJ, Ross ME, Shurtleff SA, et al: Classification, subtype discovery, and prediction of outcome in pediatric acute lymphoblastic leukemia by gene expression profiling. Cancer Cell 1: 133-143, 2002.

9. Downing JR: Acute leukemia: subtype discovery and prediction of outcome by gene expression profiling. Verh Dtsch Ges Pathol 87: 66-71, 2003.

10. Chow EJ, Simmons JH, Roth CL, et al: Increased cardiometabolic traits in pediatric survivors of acute lymphoblastic leukemia treated with total body irradiation. Biol Blood Marrow Transplant 16: 1674-1681, 2010.

11. Rahiala J, Riikonen P, Kekäläinen L and Perkkiö M: Cost analysis of the treatment of acute childhood lymphocytic leukemia according to Nordic protocols. Acta Paediatr 89: 482-487, 2000.

12. Silva A, Yunes JA, Cardoso BA, et al: PTEN posttranslational inactivation and hyperactivation of the PI3K/Akt pathway sustain primary $\mathrm{T}$ cell leukemia viability. J Clin Invest 118 3762-3774, 2008.

13. Paganin M and Ferrando A: Molecular pathogenesis and targeted therapies for NOTCH1-induced T-cell acute lymphoblastic leukemia. Blood Rev 25: 83-90, 2011.

14. Johnson GL and Lapadat R: Mitogen-activated protein kinase pathways mediated by ERK, JNK, and p38 protein kinases. Science 298: 1911-1912, 2002.
15. Pucci B, Kasten M and Giordano A: Cell cycle and apoptosis. Neoplasia 2: 291-299, 2000.

16. Krajarng A,Nilwarankoon S, Suksamrarn S and Watanapokasin R: Antiproliferative effect of $\alpha$-mangostin on canine osteosarcoma cells. Res Vet Sci 93: 788-794, 2012.

17. Elmore S: Apoptosis: a review of programmed cell death. Toxicol Pathol 35: 495-516, 2007.

18. Sun C, Guo XX, Zhu D, et al: Apoptosis is induced in cancer cells via the mitochondrial pathway by the novel xylocydine-derived compound JRS-15. Int J Mol Sci 14: 850-870, 2013.

19. Bejarano I, Redondo PC, Espino J, et al: Melatonin induces mitochondrial-mediated apoptosis in human myeloid HL-60 cells. J Pineal Res 46: 392-400, 2009.

20. Foster SS, De S, Johnson LK, Petrini JH and Stracker TH: Cell cycle- and DNA repair pathway-specific effects of apoptosis on tumor suppression. Proc Natl Acad Sci USA 109: 9953-9958, 2012.

21. Badaboina S, Bai HW, Park CH, et al: Molecular mechanism of apoptosis induction in skin cancer cells by the centipedegrass extract. BMC Complementary Altern Med 13: 350, 2013.

22. Demchenko AP: Beyond Annexin V: fluorescence response of cellular membranes to apoptosis. Cytotechnology 65: 157-172, 2013.

23. Arur S, Uche UE, Rezaul K, et al: Annexin I is an endogenous ligand that mediates apoptotic cell engulfment. Dev Cell 4: 587-598, 2003.

24. Li H, Wang P, Liu Q, Cheng X, Zhou Y and Xiao Y: Cell cycle arrest and cell apoptosis induced by Equisetum hyemale extract in murine leukemia L1210 cells. J Ethnopharmacol 144: 322-327, 2012.

25. Mohan S, Abdul AB, Abdelwahab SI, et al: Typhonium flagelliforme induces apoptosis in CEMss cells via activation of caspase-9, PARP cleavage and cytochrome c release: its activation coupled with G0/G1 phase cell cycle arrest. J Ethnopharmacol 131: 592-600, 2010.

26. Abubakar MB, Abdullah WZ, Sulaiman SA and Suen AB: A review of molecular mechanisms of the anti-leukemic effects of phenolic compounds in honey. Int J Mol Sci 13: 15054-15073, 2012.

27. Grütter MG: Caspases: key players in programmed cell death. Curr Opin Struct Biol 10: 649-655, 2000.

28. Rodriguez-Hernandez A, Brea-Calvo G, Fernandez-Ayala DJ, Cordero M, Navas P and Sanchez-Alcazar JA: Nuclear caspase-3 and caspase-7 activation, and Poly(ADP-ribose) polymerase cleavage are early events in camptothecin-induced apoptosis. Apoptosis 11: 131-139, 2006.

29. El-Mahdy MA, Zhu Q, Wang QE, Wani G and Wani AA: Thymoquinone induces apoptosis through activation of caspase- 8 and mitochondrial events in p53-null myeloblastic leukemia HL-60 cells. Int J Cancer 117: 409-417, 2005.

30. Tao Z, Zhou Y, Lu J, et al: Caspase-8 preferentially senses the apoptosis-inducing action of NG-18, a Gambogic acid derivative, in human leukemia HL-60 cells. Cancer Biol Ther 6: 691-696, 2007.

31. Granville DJ, Shaw JR, Leong S, et al: Release of cytochrome c, Bax migration, Bid cleavage, and activation of caspases 2, 3,6, 7,8, and 9 during endothelial cell apoptosis. Am J Pathol 155: $1021-1025,1999$.

32. Martinou JC and Youle RJ: Mitochondria in Apoptosis: Bcl-2 family members and mitochondrial dynamics. Dev Cell 21: 92-101, 2011.

33. Shin DY, Kim GY, Lee JH, Choi BT, Yoo YH and Choi YH: Apoptosis induction of human prostate carcinoma DU145 cells by diallyl disulfide via modulation of JNK and PI3K/AKT signaling pathways. Int J Mol Sci 13: 14158-14171, 2012.

34. Chang $\mathrm{L}$ and Karin M: Mammalian MAP kinase signalling cascades. Nature 410: 38-40, 2001.

35. Levine B, Sinha S and Kroemer G: Bcl-2 family members: dual regulators of apoptosis and autophagy. Autophagy 4: 600-606, 2008.

36. Khor TO, Gul YA, Ithnin h and Seow HF: Positive correlation between overexpression of phospho-BAD with phosphorylated Akt at serine 473 but not threonine 308 in colorectal carcinoma. Cancer Lett 210: 139-150, 2004. 